\title{
Effects of a 6-week intrinsic foot muscle exercise program on the functions of intrinsic foot muscle and dynamic balance in patients with chronic ankle instability
}

\author{
Dong-Rour Lee', Young-Eun Choi ${ }^{2}$ ** \\ 'Department of Rehabilitation Therapy Team, Good Gang-An Hospital, Busan, Korea \\ ${ }^{2}$ Department of Physical Therapy, College of Health Medicine, Kaya University, Gimhae, Korea
}

We aimed to evaluate the effects of a 6 -week intrinsic foot muscle exercise program on the activation of intrinsic foot muscle movement and dynamic balance in adults with chronic ankle stability. A total of 30 adults with chronic ankle instability were recruited. The participants were randomly assigned to a group performing intrinsic foot muscle exercises and a control group doing no exercises. We measured the activation rate and dynamic balance of the abductor hallucis, flexor digitorum brevis, flexor hallucis brevis, and quadratus plantae before and af- ter the intervention. We found that the activation rate and dynamic balance significantly increased in all intrinsic foot muscles in the experimental group. These results suggest that intrinsic foot muscle exercise for patients with chronic ankle stability is an effective treatment for improving the functions and balance ability of the intrinsic foot muscles.

Keywords: Ankle instability, Intrinsic foot muscle, Foot exercise

\section{INTRODUCTION}

Parallel with industrial development, the rate of participation in sports is rising, and this leads to increased incidence of sports injuries. Lower limb joints are the major body part that is injured during sports activities; among them, injuries most frequently occur in the ankle joint (Lynch, 2002). Ankle sprains in particular account for approximately $15 \%$ of sports-related injuries, and $40 \%$ of individuals who sprained their ankles develop chronic ankle instability (CAI) (Hootman et al., 2007; Ross and Guskiewicz, 2004). The characteristics of CAI include repeated ankle joint sprains, giving way, chronic symptoms, and reduced self-reported function (Hertel, 2002). CAI is associated with increase rates of osteoarthritis and decline of quality of life. Many patients with CAI patients cannot maintain their previous levels of physical activities; young adults with CAI walk 2000 fewer steps than healthy individuals in the same age group (Hubbard-Turner and Turner, 2015). A decrease in the physical activities and joint range of motion, as well as neuromuscular dysfunction are not only general characteristics of CAI but are also potential causes of myoatrophy (Boonyarom and Inui, 2006; Feger et al., 2015). The clinical symptoms of myoatrophy include muscle weakness, changes of motion pattern, and increased risk of injury. All these symptoms occur consistently in patients with CAI (Witchalls et al., 2012). To prevent ankle sprain, synergetic interaction between the extrinsic foot muscles as global movers and the intrinsic foot muscles (IFM) as local stabilizers of the ankle joint is critical (Mckeon et al., 2015). In particular, the IFMs are attached to tarsals, metatarsals, and phalanges that stabilize the foot at the segment level. Proper neuromuscular control of the IFMs is essential for stabilizing the foot during the stance portion of the gait cycle and for controlling motion (Kelly et al., 2014). It is a similar concept as core stability that improves global movement patterns by stabilizing the spine at the segment level. There are many ways to strengthen the IFM for sports and rehabilitation, including unilateral balance training, towel curl, short foot exercise, and toe
${ }^{*}$ Corresponding author: Young-Eun Choi (iD https://orcid.org/0000-0002-1419-8519 Department of Physical Therapy, College of Health Medicine, Kaya University, 208 Samgye-ro, Gimhae 50830, Korea

E-mail: choiye00@naver.com

Received: August 20, 2019 / Accepted: September 15, 2019
This is an Open Access article distributed under the terms of the Creative Commons Attribution Non-Commercial License (http://creativecommons.org/licenses/by-nc/4.0/) which permits unrestricted non-commercial use, distribution, and reproduction in any medium, provided the original work is properly cited. 
posture exercise (Gooding et al., 2016; Kim et al., 2013). Towel curl activates part of the IFM; however, the activation of flexor digitorum longus and flexor hallucis longus were more predominant. In contrast, short foot exercise was reported to be effective for stabilizing the foot because the IFM is exclusively contracted during this exercise (Sauer et al., 2011). Among the CAI patients, the group that maintained short foot positioning while performing balance exercise showed improvement in the self-reported function compared to a group that did not maintain short foot positioning (Sauer et al., 2010).

Posture exercises include toe spreading, hallux extension, and lesser toe extension. Previous studies comparing the activation of abductor hallucis during toe spread out and short foot exercise reported that the abductor hallucis was more activated during toe spread out (Kim et al., 2013). Recent studies have reported that toe spread, hallux extension, and lesser toe extension exercises were associated with increased activation of the plantar IFM (Fraser and Hertel, 2019; Gooding et al., 2016). Although the usage of and interest in IFM exercises is increasing in clinical practice and these exercises are effective for strengthening IFM, to the best of our knowledge, none of the studies published previously have evaluated whether toe posture exercise is effective for the rehabilitation of patients with. Therefore, our study aimed to analyze the activation of the IFM and the changes in dynamic balance in patients with CAI after a 6-week IFM exercise program.

\section{MATERIALS AND METHODS}

\section{Participants}

We recruited 30 adults with CAI (10 males, 20 females). The selection criteria for CAI were as follows: history of ankle sprain more than once with the first incidence occurring more than a year prior, experience of the ankle giving way after ankle sprain, and scores on the Cumberland ankle instability tool of 24 or below. Exclusion criteria were as follows: history of lower extremity surgery, lower extremity fractures, and immobilization of foot or ankle for $48 \mathrm{hr}$ or longer within the previous 6 months. After the objectives and contents of the study were explained, the participants voluntarily participated in the study after written consent was obtained. The 30 participants were randomly assigned to the experimental group performing IFM exercises and a control group doing no exercises. The study procedures were approved by the Research Ethics Committee of Kaya University (approval number: 20180187).

\section{Ultrasonography}

Ultrasonography equipment (ultrasonography, Prosound 2, Hitachi Aloka Medical, Tokyo, Japan) was used to measure the thickness of the IFMs. We used a 6-13 MHz linear transducer probe. To measure the abductor hallucis, the participants adopted a supine position with their hip externally rotated and a knee slightly flexed. The probe was positioned on the origin of a muscle above medial calcaneal tuberosity towards the navicular tuberosity. The thickest part of the muscle was determined using longitudinal scanning, and the transducer was moved proximally from navicular tuberosity by $1-$ to $2-\mathrm{cm}$ increments. A still frame was then captured, and the muscle thickness was measured. To measure the flexor hallucis brevis, flexor digitorum brevis, and quadratus plantae, the participants lay prone with their foot hanging freely off the bed. To measure the thickness of the flexor digitorum brevis, the proximal to distal 1st metatarsal and the thickest part of muscle belly was longitudinally scanned. A still frame was captured, and the muscle thickness was measured. To measure the thickness of the flexor digitorum brevis, the ankle with muscle insertion was longitudinally scanned along the line connecting the medial tuberosity and the third toe on the plantar surface. After determining the thickest part of the muscle belly, a still frame was captured, and the muscle thickness was measured. The quadratus plantae was located in the deep layer under the flexor digitorum brevis. Longitudinal scanning was performed along the sagittal plane of the talocalcaneonavicular joint, and the thickest part of the muscle belly was determined. A still frame was then captured, and the muscle thickness was measured (Mickle et al., 2013). Muscle thickness was measured during IFM exercises and at rest and the activation ratio was calculated using the following equation: contracted measurement/resting measurement.

An activation ratio $>1$ indicates increased muscle thickness and activation ratio $<1$ indicates decreased muscle thickness (Pulkovski et al., 2012; Teyhen et al., 2005).

\section{Dynamic balance test}

The star excursion balance test (SEBT) evaluates dynamic balance in the one-leg standing position. While barefoot, the starting posture is to place both hands on the pelvis and stand on one foot. Then, the opposite foot is stretched out such that the tip of the toe can touch the ground. The maximum distance that allows return to the starting posture was measured in centimeters. The maximum distance was measured from three directions: anterior, posteromedial, and posterolateral (Gribble et al., 2012). Prior to the test, the examiner demonstrated to the participants and the test was per- 

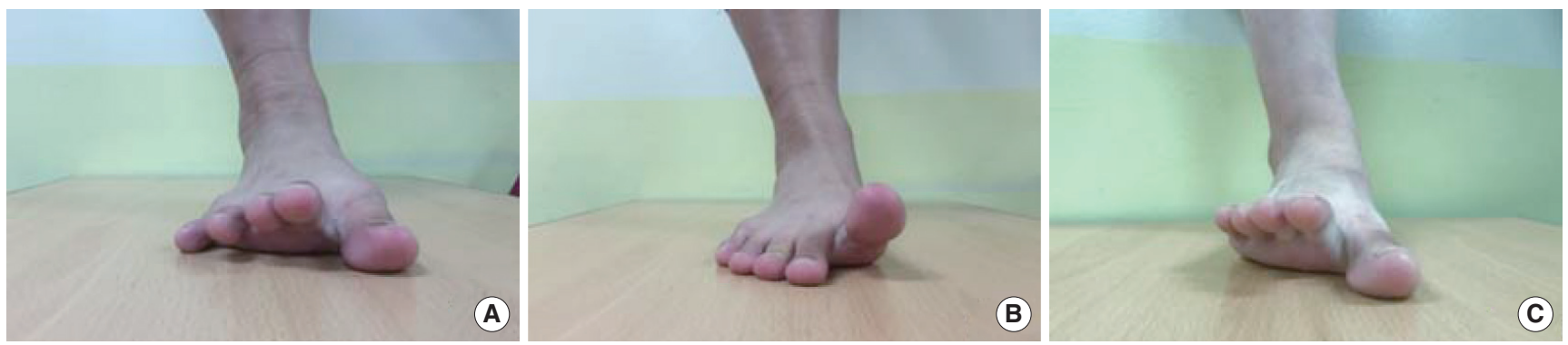

Fig. 1. Intrinsic foot muscle exercises. (A) Toe spread out. (B) First toe extension. (C) Second to fifth toe extension.

Table 1. Intrinsic foot muscle exercise program

\begin{tabular}{llcc}
\hline Week & Progression & Contraction time $(\mathrm{sec})$ & Sets $\times$ repetitions \\
\hline 1,2 & Sitting & $3 / 8 / 20$ & $4 \times 15 / 4 \times 8 / 4 \times 3$ \\
3,4 & Standing & $3 / 8 / 20$ & $4 \times 15 / 4 \times 8 / 4 \times 3$ \\
5,6 & One-leg standing & $3 / 8 / 20$ & $4 \times 15 / 4 \times 8 / 4 \times 3$
\end{tabular}

formed after the test method was fully understood. Because there were practice effects before participants became accustomed to the test method, the test was conducted after 4 practice rounds. Then, the mean distance was obtained after three trials. The maximum distance was affected by not only balance ability but also by leg length. To exclude this effect, the measured distance was divided by leg length and multiplied by 100 such that the maximum distance was calculated as \% leg length. After obtaining the sum of right and left sides, the mean value was calculated.

\section{Intrinsic foot muscle exercise program}

The participants took part in an intrinsic foot muscle exercise program three times per week for 6 weeks under supervision. Intrinsic foot muscle training includes toe spread out, first toe extension, and second to fifth toe extension exercise (Fig. 1). Toe spread out exercise was performed by extending all toes and then pushing the fifth toe down toward lateral direction and pushing the first toe down toward medial direction. Each exercise was performed with 104 repetitions, and the time spent while maintaining contraction was controlled according to the number of repetitions weeks (Fraser and Hertel, 2019; Long et al., 2013). The exercise was performed in a sitting position for the first 2 weeks and in a standing position on the $3 \mathrm{rd}$ and 4 th weeks. The exercise was performed in a one-leg standing position during the 5th and 6th (Table 1).

\section{Statistical analysis}

To analyze general characteristics of the participants, descriptive statistics was used to obtain means and standard deviations. The Shapiro-Wilk test was used to analyze normal distribution of each
Table 2. General characteristics of the subjects $(n=30)$

\begin{tabular}{lcccc}
\hline Group & Age $(\mathrm{yr})$ & Height $(\mathrm{cm})$ & Weight $(\mathrm{kg})$ & Sex, male:female \\
\hline Experimental & $20.85 \pm 1.09$ & $166.42 \pm 11.34$ & $62.14 \pm 14.80$ & $5: 10$ \\
Control & $20.80 \pm 0.86$ & $167.40 \pm 10.60$ & $67.00 \pm 24.13$ & $5: 10$ \\
\hline
\end{tabular}

Values are presented as mean \pm standard deviation.

measured item, and the normality of collected data was determined. Independent $t$-tests were conducted to test homogeneity between the groups. Paired $t$-tests were conducted to examine the differences before and after exercise in each group. The independent $t$-test was also used for comparisons between groups. The reliability of muscle thickness measurement within the participants was analyzed using intraclass correlation coefficients. All statistical analyses were conducted using IBM SPSS ver. 18.0 (IBM Co., Armonk, NY, USA). The level of significance was $\alpha=0.05$.

\section{RESULTS}

\section{General characteristics}

This study divided the total of 30 subjects with CAI into experimental group with intrinsic foot muscle exercises and no exercise control. The general characteristics of the two groups are shown in Table 2.

\section{Reliability of the ultrasonography measurement}

The confidence range for muscle-specific contraction ratio changes according to toe posture was $0.77-0.96$ and the standard error for measurement was $0.06-0.15$ (Table 3).

\section{Effects of IFM exercises on the muscle activation after intervention}

All the abductor hallucis, flexor digitorum brevis, flexor hallucis brevis, quadratus plantae muscles increased in toe spread out after 6 weeks of exercise, but only the flexor digitorum brevis, flexor hallucis brevis, quadratus plantae muscles increased in first 
toe extension and second to fifth toe extension (Table 4).

\section{Effects of IFM exercises on the dynamic balance after intervention}

The experimental group showed significant difference in com-

Table 3. Reliability of measurements of muscle's contraction ratio according to postures

\begin{tabular}{llll}
\hline Variable & ICC & $95 \%$ Cl & SEM \\
\hline Toes spread out & & & \\
Abductor hallucis & 0.93 & $0.86-0.95$ & 0.08 \\
Flexor digitorum brevis & 0.80 & $0.65-0.91$ & 0.14 \\
Flexor hallucis brevis & 0.79 & $0.62-0.88$ & 0.15 \\
$\quad$ Quadratus plantae & 0.87 & $0.77-0.92$ & 0.11 \\
First-toe-extension & & & \\
Abductor hallucis & 0.96 & $0.93-0.98$ & 0.06 \\
Flexor digitorum brevis & 0.79 & $0.64-0.89$ & 0.15 \\
Flexor hallucis brevis & 0.77 & $0.60-0.88$ & 0.15 \\
$\quad$ Quadratus plantae & 0.83 & $0.70-0.91$ & 0.13 \\
Second-to fifth-toe extension & & & \\
$\quad$ Abductor hallucis & 0.91 & $0.83-0.95$ & 0.10 \\
Flexor digitorum brevis & 0.78 & $0.62-0.88$ & 0.15 \\
Flexor hallucis brevis & 0.80 & $0.65-0.90$ & 0.14 \\
$\quad$ Quadratus plantae & 0.88 & $0.78-0.93$ & 0.11
\end{tabular}

ICC, intraclass correlation coefficients; $\mathrm{Cl}$, confidence interval; SEM, standard error of measurement. posite reach distance, the control group showed no significant difference. The change was significantly higher in the experimental group compared to the control group (Table 5).

\section{DISCUSSION}

Ankle sprain has a high recurrence rate, exceeding $70 \%$. It can develop into CAI. One of the factors causing CAI is changes in IFM activation. In the present study, we implemented an IFM exercise program using toe posture exercise for patients with CAI and we examined the activation of IFM and changes of balance. After 6 weeks of intervention, the abductor hallucis, flexor digitorum brevis, flexor hallucis brevis, and quadratus plantae all showed increased activation. After implementing short foot and toe spread out exercises in participants with mild hallux valgus,

Table 5. Comparison of dynamic balance before and after intrinsic foot muscle exercises

\begin{tabular}{lccc}
\hline $\begin{array}{l}\text { Composite reach distance } \\
\text { (\% leg length) }\end{array}$ & Experimental & Control & $P$-value \\
\hline Pre & $66.79 \pm 9.64$ & $65.44 \pm 8.67$ & 0.29 \\
Post & $70.94 \pm 8.74$ & $66.74 \pm 9.09$ & 0.00 \\
Post-pre & $6.10 \pm 2.34$ & $2.30 \pm 1.78$ & 0.00 \\
$P$-value & 0.00 & 0.12 & \\
\hline
\end{tabular}

Values are presented as mean \pm standard deviation.

Table 4. Comparison of muscle activation before and after intrinsic foot muscle exercises

\begin{tabular}{|c|c|c|c|c|c|c|c|}
\hline \multirow{2}{*}{ Variable } & \multicolumn{3}{|c|}{ Experimental } & \multicolumn{3}{|c|}{ Control } & \multirow{2}{*}{$P$-value } \\
\hline & Pre & Post & Post-pre & Pre & Post & Post-pre & \\
\hline \multicolumn{8}{|l|}{ TSO } \\
\hline $\mathrm{AbH}$ & $1.05 \pm 0.12$ & $1.16 \pm 0.10^{*}$ & $0.10 \pm 0.07$ & $1.00 \pm 0.69$ & $1.01 \pm 0.09$ & $0.00 \pm 0.48$ & 0.00 \\
\hline FDB & $1.05 \pm 0.18$ & $1.14 \pm 0.18$ & $0.08 \pm 0.19$ & $1.13 \pm 0.17$ & $1.10 \pm 0.15$ & $-0.05 \pm 0.06$ & 0.01 \\
\hline FHB & $1.13 \pm 0.23$ & $1.19 \pm 0.26^{*}$ & $0.13 \pm 0.12$ & $1.14 \pm 0.24$ & $1.13 \pm 0.22$ & $-0.01 \pm 0.12$ & 0.00 \\
\hline $\mathrm{QP}$ & $1.05 \pm 0.15$ & $1.11 \pm 0.14^{*}$ & $0.08 \pm 0.07$ & $1.05 \pm 0.17$ & $1.08 \pm 0.15$ & $0.02 \pm 0.09$ & 0.05 \\
\hline \multicolumn{8}{|l|}{ FIE } \\
\hline $\mathrm{AbH}$ & $1.07 \pm 0.13$ & $1.15 \pm 0.12^{*}$ & $0.07 \pm 1.11$ & $1.01 \pm 0.12$ & $1.03 \pm 0.14$ & $0.01 \pm 0.06$ & 0.12 \\
\hline FDB & $1.04 \pm 0.16$ & $1.19 \pm 0.18^{*}$ & $0.11 \pm 0.14$ & $1.13 \pm 0.18$ & $1.13 \pm 0.16$ & $-0.02 \pm 0.09$ & 0.00 \\
\hline FHB & $1.16 \pm 0.23$ & $1.20 \pm 0.25$ & $0.11 \pm 0.14$ & $1.13 \pm 0.15$ & $1.11 \pm 0.19$ & $-0.02 \pm 0.09$ & 0.00 \\
\hline $\mathrm{QP}$ & $1.03 \pm 0.14$ & $1.07 \pm 0.17$ & $0.08 \pm 0.11$ & $1.04 \pm 0.17$ & $1.02 \pm 0.18$ & $-0.01 \pm 0.10$ & 0.01 \\
\hline \multicolumn{8}{|l|}{ SFTE } \\
\hline $\mathrm{AbH}$ & $1.09 \pm 0.12$ & $1.16 \pm 0.11$ & $0.06 \pm 0.10$ & $1.00 \pm 0.10$ & $1.00 \pm 0.12$ & $0.00 \pm 0.06$ & 0.05 \\
\hline FDB & $1.05 \pm 0.13$ & $1.20 \pm 0.18^{*}$ & $0.14 \pm 0.11$ & $1.11 \pm 0.14$ & $1.11 \pm 0.15$ & $-0.00 \pm 0.05$ & 0.00 \\
\hline FHB & $1.16 \pm 0.27$ & $1.23 \pm 0.29 *$ & $0.11 \pm 0.08$ & $1.13 \pm 0.19$ & $1.15 \pm 0.22$ & $0.02 \pm 0.12$ & 0.03 \\
\hline $\mathrm{QP}$ & $1.05 \pm 0.13$ & $1.09 \pm 0.18$ & $0.08 \pm 0.13$ & $1.06 \pm 0.13$ & $1.05 \pm 0.17$ & $-0.00 \pm 0.08$ & 0.03 \\
\hline
\end{tabular}

Values are presented as mean \pm standard deviation.

TSO, toe spread out; AbH, abductor hallucis; FDB, flexor digitorum brevis; FHB, flexor hallucis brevis; QP, quadratus plantae; FTE, first toe extension; SFTE, second to fifth toe extension.

${ }^{*} P<0.05$, statistically significant different between pre and post. 
the activation of abductor halluces was compared using surface electromyography. The result showed that the activation of the abductor hallucis was higher after toe spread out exercise than after short foot exercise (Kim et al., 2013). Although comparing the level of activation according to exercise methods was not part of the objectives of our study, our results showed that activation of the abductor hallucis was greater when toe spread out exercise was performed after the IFM exercise program. Toe spread out exercise utilizes the motion of first and fifth toe returning to the ground from flexion after toe extension and abduction. Therefore, the activation of the abductor halluces could have been increased. A study comparing the cross-sectional changes of muscles during toe posture exercise and resting in healthy adults reported that the activation of the hallucis brevis and abductor hallucis were increased after first toe extension by $18 \%$ and $17 \%$, respectively, compared to resting (Gooding et al., 2016). It is presumed that these two muscles were activated to stabilize the first metatarsal head during the first toe extension. In contrast, in a study comparing the changes of intrinsic muscle activation after 4-week IFM exercise, no significant difference was observed in intrinsic muscle activation after the intervention; muscle activity was rather decreased, contradicting our present results (Fraser and Hertel, 2019). As the exercise program was performed under the supervision of a therapist, it is likely that more accurate practice and feedback were available during the exercise. Furthermore, because our intervention period was longer by 2 weeks, we presume that the muscle synergistic pattern for motor tasks was decreased and contraction of the individual muscles was enabled.

Measurement of dynamic balance using SEBT showed that dynamic balance was significantly greater after the exercise. Short foot exercise, widely believed to be an IFM strengthening exercise, is used as a sensorimotor training technique for improving proprioception as well as postural alignment and postural stability (Sauer et al., 2011). While applying the balance training program for 4 weeks in healthy individuals, the group who performed short foot exercise was compared to a control group. Static balance improved, though not significantly (Rothermel et al., 2004). This was different from our study results; however the improvement of balance after exercise is an important result because the participants were healthy individuals without balance deficit. Another study compared patients with functional ankle instability to healthy individuals before and after the application of functional balance training program. Postural stability improved in all patients after performing short foot exercise during the training period (Sauer et al., 2010). In the present study, unlike implement- ing IFM training during ankle stability balance training, there was improvement in dynamic balance after IFM exercise. In previous studies, it was not clear whether the improvement of dynamic balance was due to strengthening of IFM. Our study results suggest that improvement of IFM functions leads to changes in postural stability.

Our study results suggest that an IFM exercise program using toe posture provides foot stability in patients with CAI by improving the functions and dynamic balance of IFM. There are some limitations of our study. The number of participants was small and the participants were relatively young. Further studies must be conducted to overcome such limitations. Moreover, studies wherein the program is further developed and used for patients who require IFM strengthening in clinical practice are also needed.

\section{CONFLICT OF INTEREST}

No potential conflict of interest relevant to this article was reported.

\section{REFERENCES}

Boonyarom O, Inui K. Atrophy and hypertrophy of skeletal muscles: structural and functional aspects. Acta Physiol (Oxf) 2006;188:77-89.

Feger MA, Donovan L, Hart JM, Hertel J. Lower extremity muscle activation in patients with or without chronic ankle instability during walking. J Athl Train 2015;50:350-357.

Fraser JJ, Hertel J. Effects of a 4-week intrinsic foot muscle exercise program on motor function: a preliminary randomized control trial. J Sport Rehabil 2019;28:339-349.

Gooding TM, Feger MA, Hart JM, Hertel J. Intrinsic foot muscle activation during specific exercises: a T2 time magnetic resonance imaging study. J Athl Train 2016;51:644-650.

Gribble PA, Hertel J, Plisky P. Using the Star Excursion Balance Test to assess dynamic postural-control deficits and outcomes in lower extremity injury: a literature and systematic review. J Athl Train 2012;47:339357.

Hertel J. Functional anatomy, pathomechanics, and pathophysiology of lateral ankle instability. J Athl Train 2002;37:364-375.

Hootman JM, Dick R, Agel J. Epidemiology of collegiate injuries for 15 sports: summary and recommendations for injury prevention initiatives. J Athl Train 2007;42:311-319.

Hubbard-Turner T, Turner MJ. Physical activity levels in college students with chronic ankle instability. J Athl Train 2015;50:742-747.

Kelly LA, Cresswell AG, Racinais S, Whiteley R, Lichtwark G. Intrinsic 
foot muscles have the capacity to control deformation of the longitudinal arch. J R Soc Interface 2014;11:20131188.

Kim MH, Kwon OY, Kim SH, Jung DY. Comparison of muscle activities of abductor hallucis and adductor hallucis between the short foot and toe-spread-out exercises in subjects with mild hallux valgus. J Back Musculoskelet Rehabil 2013;26:163-168.

Long L, Jackson K, Lauach LL. A home-based exercise program for the foot and ankle to improve balance, muscle performance and flexibility in community dwelling older adult: a pilot study. Int J Phys Med Rehabil 2013;1:120.

Lynch SA. Assessment of the injured ankle in the athlete. J Athl Train 2002;37:406-412.

McKeon PO, Hertel J, Bramble D, Davis I. The foot core system: a new paradigm for understanding intrinsic foot muscle function. Br J Sports Med 2015;49:290.

Mickle KJ, Nester CJ, Crofts G, Steele JR. Reliability of ultrasound to measure morphology of the toe flexor muscles. J Foot Ankle Res 2013;6:12.

Pulkovski N, Mannion AF, Caporaso F, Toma V, Gubler D, Helbling D, Sprott $\mathrm{H}$. Ultrasound assessment of transversus abdominis muscle contraction ratio during abdominal hollowing: a useful tool to distinguish between patients with chronic low back pain and healthy con- trols? Eur Spine J 2012;21 Suppl 6:S750-759.

Ross SE, Guskiewicz KM. Examination of static and dynamic postural stability in individuals with functionally stable and unstable ankles. Clin J Sport Med 2004;14:332-338.

Rothermel SA, Hale SA, Hertel J, Denegar CR. Effect of active foot positioning on the outcome of a balance training program. Phys Ther Sport 2004;5:98-103.

Sauer LD, Beazell J, Hertel J. Considering the intrinsic foot musculature in evaluation and rehabilitation for lower extremity injuries: a case review. Athl Train Sports Health Care 2011;3:43-47.

Sauer LD, Saliba SA, Ingersoll CD, Kerrigan DC, Pietrosimone BP, Hertel J. Effects of rehabilitation incorporating short foot exercises on self-reported function, static and dynamic balance in chronic ankle instability patients. J Athl Train 2010;45:S67.

Teyhen DS, Miltenberger CE, Deiters HM, Del Toro YM, Pulliam JN, Childs JD, Boyles RE, Flynn TW. The use of ultrasound imaging of the abdominal drawing-in maneuver in subjects with low back pain. J Orthop Sports Phys Ther 2005;35:346-355.

Witchalls J, Blanch P, Waddington G, Adams R. Intrinsic functional deficits associated with increased risk of ankle injuries: a systematic review with meta-analysis. Br J Sports Med 2012;46:515-523. 